\title{
Prognostic value of changes in arterial stiffness in men with coronary artery disease
}

\author{
This article was published in the following Dove Press journal: \\ Vascular Health and Risk Management \\ 3 November 2010 \\ Number of times this article has been viewed
}

\section{lana A Orlova \\ Eradzh Yu Nuraliev \\ Elena B Yarovaya \\ Fail T Ageev}

Outpatient department, Russian Cardiology Research Center, Moscow, Russian Federation
Correspondence : lana A Orlova Outpatient department, Russian Cardiology Research Center, 3rd Cherepkovskaya Street I5A, Moscow 121552, Russia

Tel +7 4954146970

Fax +74954I466 I2

E-mail orlova@cardio.ru
Background: Men with coronary artery disease (CAD) have been shown to have enhanced arterial stiffness. Arterial function may change over time according to treatment, but the prognostic value of these changes has not been investigated.

Objectives: The aim of the present study was to assess whether an improvement in large artery rigidity in response to treatment, could predict a more favorable prognosis in a population of men with CAD.

Methods: A total of 161 men with CAD (mean age $56.8 \pm 10.9$ years) being treated with conventional therapy underwent brachial-ankle pulse wave velocity (PWVba) measurements at baseline and after six months. Follow-up period was 3.5 years. End-points were major adverse cardiac events (MACE): acute myocardial infarction, unstable angina, coronary intervention, or cardiac death.

Results: During the three-year follow-up period (since initial six-month follow-up), 30 patients experienced MACE. After six-month follow-up, PWVba had not improved ( $\triangle \mathrm{PWVba} \geq 0 \%$, relative to baseline) in $85(52.8 \%)$ of 161 men (Group 1), whereas it had improved $(\triangle \mathrm{PWVba}<0 \%)$ in the remaining 76 men $(47.2 \%)$ (Group 2). During follow-up, we noticed 24 cardiovascular events in Group 1 and six events in Group $2(P<0.001)$. Cox proportional hazards analyses demonstrated that independent of conventional risk factor changes, absence of PWVba decrease was a predictor of MACE (RR 3.99; 95\% CI:1.81-8.78; $P=0.004$ ). The sensitivity of $\triangle \mathrm{PWVba}$ was $80 \%$ and its specificity was $54 \%$.

Conclusions: This study demonstrates that an improvement in arterial stiffness may be obtained after six months of conventional therapy and clearly identifies patients who have a more favorable prognosis.

Keywords: arterial stiffness, coronary artery disease, prognosis

\section{Introduction}

Elevated arterial stiffness has been associated with progression of cardiovascular morbidity and mortality due to arterial hypertension, end-stage renal failure, diabetes mellitus, and other conditions. ${ }^{1-3}$ Data exist to show that decreased arterial elasticity is a predictor of future development of cardiovascular complications in patients with coronary artery disease (CAD). ${ }^{4,5}$ In order to consider increased arterial stiffness as something necessitating therapeutic focus, crucial data has been acquired showing that decreasing arterial rigidity leads directly to a decreased risk of major adverse cardiac events (MACE). Obtaining such confirmation was the purpose of this work. 


\section{Methods}

\section{Patients}

After giving informed consent, ambulatory patients aged 35-75 years, with proven clinically stable coronary artery disease, were considered for the study. Eligible participants were recruited by the outpatient department of the Russian Cardiology Research Center in Moscow if they met at least one of the following inclusion criteria: 1) history of myocardial infarction; 2) angiographic evidence of at least $50 \%$ stenosis in at least one coronary artery; 3 ) evidence of exercise-induced ischemia on treadmill electrocardiogram or stress nuclear perfusion imaging; or 4) history of coronary revascularization.

The study began in September 2004 and recruitment was completed in September 2005. The mean \pm SD for follow-up was $586 \pm 461$ days. Of the 224 consecutive patients initially screened, 161 remained on the study for our 6-month follow-up examination. All 161 patients were white males, with a $12 \%$ prevalence of diabetes mellitus (Table 1). The mean \pm SD age of the cohort at inclusion was $56.8 \pm 10.9$ years. Major exclusion criteria included: acute myocardial infarction (MI) or stroke within the past three months; percutaneous coronary intervention (PCI), coronary-artery bypass grafting (CABG), implantation, or planned implantation of a cardioverter defibrillator or biventricular pacemaker within the past three months; clinically significant, uncorrected primary valvular heart; hypertrophic cardiomyopathy; systemic disease; acute or chronic liver disease; plasma creatinine level $>2.5 \mathrm{mg} / \mathrm{dL}$; current presence of clinical signs of heart failure, and ejection fraction below $40 \%$ as assessed locally by echocardiography or another method if echocardiography was technically impossible;

Table I Characteristics of studied population $(n=161)$

Age, years (mean $\pm S D$ ) $56.8 \pm 10.9$

Patients with a previous $\mathrm{MI}(\%)$

Patients with angina pectoris (\%)

Patients with hypertension (\%)

Current smokers (\%)

Patients with DM (\%)

Medication

Acetylsalicylic acid and/or clopidogrel (\%)

Nitrates (\%)

$\beta$-blockers (\%)

Calcium channel blockers (\%)

ACEI or ARB (\%)

Statins (\%)

$101(62.7 \%)$

$113(70.2 \%)$

$113(70.2 \%)$

$48(29.8 \%)$

$20(12.4 \%)$

I4I (87.6\%)

$27(16.8 \%)$

$132(82.0 \%)$

$30(18.6 \%)$

$93(57.8 \%)$

$133(82.6 \%)$

Abbreviations: SD, standard deviation; MI, myocardial infarction; DM, diabetes mellitus; ACEI, angiotensin-converting enzyme inhibitor; ARB, angiotensin II receptor blockers. diabetes mellitus (DM) requiring medication; any other condition that would substantially reduce life expectancy or limit compliance with the protocol.

All patients received conventional therapy. Characteristics of the studied population are reported in Table 1.

\section{Data collection}

Information compiled from the questionnaire filled out at inclusion included: personal and family histories; smoking habits; and prior history of cardiovascular disease, including cardiac insufficiency, peripheral vascular disease, and cerebrovascular disease. Twice - at baseline and after six months - blood pressure (BP), fasting blood glucose (FBG), serum total cholesterol (TC), triglycerides (TG), body mass index (BMI), arterial stiffness, and other risk factors were assessed. BP was measured in the right arm after five minutes of recumbence using a mercury sphygmomanometer and cuff of appropriate size. Phases I and V of the Korotkoff sounds were taken as the systolic BP (SBP) and diastolic BP (DBP) values respectively. The mean $\mathrm{BP}(\mathrm{MBP})$ was calculated as follows: $\mathrm{MBP}=\mathrm{DBP}+[(\mathrm{SBP}-\mathrm{DBP}) / 3]$. Three measurements were made at two-minute intervals; the last two measurements were averaged and considered to be representative. The patient's heart rate was determined from the three-lead orthogonal ECG. Arterial stiffness was assessed using the brachial-ankle pulse wave velocity (PWVba) with a VaSera VS-1000 device (Fukuda Denshi, Tokyo, Japan). ${ }^{6,7}$ Measurements were made according to the recommendations for user procedures. ${ }^{8}$ The PWVba was measured at inclusion and again after six months. The change in PWVba ( $\triangle \mathrm{PWVba})$, measured in meters per second $(\mathrm{m} / \mathrm{s})$ and used as a prognostic variable, was quantified as follows: $\triangle \mathrm{PWVba}=(\mathrm{PWVba}$ after six months $)-($ PWVba at inclusion).

\section{Follow-up}

The patients were followed every six months. Upon follow-up, patients were asked to fill out a questionnaire regarding hospitalizations and outpatient clinic visits in the preceding six months. ACE of interest for this study were acute myocardial infarction, unstable angina, coronary intervention, or cardiac death. When a possible event was recorded by the participant, hospital discharge letters, results of laboratories, and other relevant examinations were collected.

\section{Analyses}

The primary analysis concerned the survival curves and Cox proportional hazards model. Survival was estimated by the Kaplan-Meier product-limit method and compared using 
the Mantel (log-rank) test. Factors prognostic of survival were identified with use of the Cox proportional hazards regression model. The assumption of proportional hazards over time was verified before the analyses and was met by all covariates. The assumption concerning linearity of continuous covariates was also verified before analysis. Multivariate Cox modeling was the primary statistical analysis used to determine the independent relationship of PWV changes and other baseline characteristics with survival. Variables were considered to be prognostic when they were found to be statistically significant in the Cox proportional hazards regression model $(P<0.05$, adjusted for all variables retained in the final model). Adjusted hazard rate ratios (RR) were calculated as the antilogarithm of the $\beta$ coefficient of the Cox proportional hazards regression of the outcome events, with different variables entered in the models. The $95 \%$ confidence intervals (CI) for the adjusted RR estimates were obtained using the following formula: antilogarithm $(\beta \pm 1.96 \mathrm{SE})$, where SE is the standard error of $\beta$.

To assess $\triangle \mathrm{PWV}$ as a prognostic variable test with the use of receiver operating characteristic (ROC) curves, we calculated sensitivities, specificities, positive predictive values, and negative predictive values to predict mortality at different cutoff values. Optimal PWV cutoff values were defined as the maximization of the sum of sensitivity and specificity.

Data are expressed as mean \pm SD for normally distributed variables or median (lowest quartile; highest quartile) for not normally distributed variables. ANOVA was used for comparison of normally distributed variables and in the alternative situation, the non parametric equivalent Kruskal-Willis test was used. Differences in frequency were tested by $\chi^{2}$ analysis. All tests were two-sided, and analyses were performed using SPSS (v. 14.0;SPSS, Chicago, IL) and STATISTICA (v. 6.0; Statsoft, Tulsa. OK) software. A value of $P<0.05$ was considered significant.

\section{Results}

In the studied cohort, from six months until the end of the study, we recorded 30 cardiovascular complications: six MI, 11 PCI and CABG, 10 hospitalizations due to unstable angina, and three cardiac deaths. Patients who experienced MACE in the first six months of observation were not included in our analyses. All investigating parameter changes, from inclusion until six months time, are reported in Table 2. For the first six months, only diastolic BP and MAP showed a small (non significant) decrease, while all other parameters remained unchanged.
Table 2 Changes in investigating parameters from inclusion until six months of follow-up in entire cohort

\begin{tabular}{llll}
\hline & $\begin{array}{l}\text { Baseline } \\
\text { (mean } \pm \text { SD) }\end{array}$ & $\begin{array}{l}\text { Six months } \\
\text { (mean } \pm \text { SD) }\end{array}$ & P-value \\
\hline PWVba $(\mathrm{m} / \mathrm{s})$ & $13.60 \pm 2.44$ & $13.75 \pm 2.27$ & 0.315 \\
SBP $(\mathrm{mmHg})$ & $135.2 \pm 19.2$ & $135.9 \pm 14.9$ & 0.431 \\
DBP $(\mathrm{mmHg})$ & $87.4 \pm 11.5$ & $86.6 \pm 10.2$ & 0.060 \\
PP $(\mathrm{mmHg})$ & $48.9 \pm 12.0$ & $49.6 \pm 11.8$ & 0.152 \\
MAP $(\mathrm{mmHg})$ & $103.0 \pm 13.4$ & $102.7 \pm 12.1$ & 0.088 \\
FBG $(\mathrm{mmol} / \mathrm{L})$ & $5.98 \pm 1.36$ & $5.75 \pm 1.00$ & 0.203 \\
TC $(\mathrm{mmol} / \mathrm{L})$ & $5.07 \pm 1.29$ & $5.10 \pm 1.14$ & 0.428 \\
TG $(\mathrm{mmol} / \mathrm{L})$ & $1.33(0.97 ; 1.91)^{*}$ & $1.23(0.94 ; 18 \mathrm{I}) *$ & 0.139 \\
\hline
\end{tabular}

Note: *TG values are the median (lowest quartile; highest quartile).

Abbreviations: SD, standard deviation; PWVba, brachial-ankle pulse wave velocity; SBP, systolic blood pressure; DBP, diastolic blood pressure; PP, pulse pressure; MAP, mean arterial pressure; FBG, fasting blood glucose; TC, serum total cholesterol; TG, triglycerides.

The $\triangle \mathrm{PWV}$ was correlated with changes in MAP $(r=0.36 ; P<0.001)$, SBP $(r=0.32 ; P<0.001)$, DBP ( $r=0.32 ; P<0.001)$, but not to $\Delta \mathrm{FGB}, \Delta \mathrm{TC}$, or $\Delta \mathrm{TG}$.

Patients were separated into two groups based on the direction of change in the stiffness of their arterial walls. The ROC curve showed that the cutoff value for $\triangle \mathrm{PWV}$ was $0.05 \mathrm{~m} / \mathrm{s}$ (ie, $\cong 0$ ). Area under the curve was $0.62 \pm 0.05$ $(P=0.032)$. In six months, $\mathrm{PWVba}$ decreased in 76 patients (Group 1) and in the other 85 patients, either an increase or no change was observed (Group 2). In patients who were observed to have improved mechanical characteristics of their large arteries in the first six months, there were six MACE, while the others had 24 MACE. In our analyses, an increased or unchanged PWVba was defined as "positive" and a decrease in PWVba was considered "negative". Characteristics of patients in each group are provided in Table 3.

In patients with a demonstrated decrease in PWVba at six months (Group 1), it was shown that there was a higher baseline PWVba $14.19 \pm 2.40 \mathrm{~m} / \mathrm{s}$, compared with $13.15 \pm 2.16 \mathrm{~m} / \mathrm{s}(P=0.004)$ in Group 2 .

For 70 of 76 patients in Group 1, there were no MACE in the last three years of the study, while 24 out of 85 patients in Group 2 had complications. The sensitivity of $\triangle \mathrm{PWV}$ was $80 \%$ and its specificity was $54 \%$.

Thus, with the aid of univariate Cox proportional hazard regression analysis, we studied the influence of given parameters, at the six-month follow-up, on the occurrence of MACE in the next three years. Absence of arterial stiffness decrease was a predictor of MACE. Increase, or absence of change in PWVba (positive $\triangle \mathrm{PWVba}$ ), increased the risk of MACE in the following three years by nearly four times (RR 3.77; 95\% CI:1.83-7.77; $\mathrm{t}=3.02, P=0.003$ ). 
Table 3 Characteristics of patients in groups with "negative" and "positive" PWVba dynamics for the first six months of observation $(n=161)$

\begin{tabular}{|c|c|c|c|}
\hline & $\begin{array}{l}\Delta \mathrm{PWVba}<0 \\
(\mathrm{n}=76)\end{array}$ & $\begin{array}{l}\Delta P W V b a \geq 0 \\
(n=85)\end{array}$ & $P$-value \\
\hline $\begin{array}{l}\text { Patients with history } \\
\text { of MACE (\%) }\end{array}$ & $6(7.9 \%)$ & $24(28.2 \%)$ & 0.001 \\
\hline Age (mean years $\pm S D$ ) & $56.9 \pm 10.8$ & $56.8 \pm 11.0$ & 0.649 \\
\hline $\begin{array}{l}\text { Patients with a } \\
\text { previous MI (\%) }\end{array}$ & $46(60.5 \%)$ & $55(64.7 \%)$ & 0.626 \\
\hline $\begin{array}{l}\text { Patients with } \\
\text { hypertension (\%) }\end{array}$ & $53(69.7 \%)$ & $60(70.6 \%)$ & 1.000 \\
\hline $\begin{array}{l}\text { Patients with angina } \\
\text { pectoris (\%) }\end{array}$ & $52(68.4 \%)$ & 61 (7I.7\%) & 0.731 \\
\hline Current smokers (\%) & $23(30.3 \%)$ & 25 (29.4\%) & 1.000 \\
\hline Patients with DM (\%) & $12(15.8 \%)$ & $8(9.4 \%)$ & 0.242 \\
\hline $\mathrm{SBP}(\mathrm{mmHg})$ & $139.1 \pm 19.4$ & $133.0 \pm 18.8$ & 0.046 \\
\hline $\mathrm{DBP}(\mathrm{mmHg})$ & $90.0 \pm 11.5$ & $85.8 \pm 11.8$ & 0.023 \\
\hline $\mathrm{MAP}(\mathrm{mmHg})$ & $106.4 \pm 13.4$ & $101.5 \pm 13.4$ & 0.024 \\
\hline BMI $\left(\mathrm{kg} / \mathrm{m}^{2}\right)$ & $27.6 \pm 3.6$ & $27.3 \pm 4.0$ & 0.538 \\
\hline $\mathrm{FBG}(\mathrm{mmol} / \mathrm{L})$ & $5.95 \pm 1.36$ & $5.76 \pm 0.92$ & 0.320 \\
\hline $\mathrm{TC}(\mathrm{mmol} / \mathrm{L})$ & $5.32 \pm 1.39$ & $4.88 \pm 1.26$ & 0.042 \\
\hline $\mathrm{TG}(\mathrm{mmol} / \mathrm{L})$ & $1.26(1.03 ; 1.77)$ & $1.37(0.90 ; 1.35)$ & 0.836 \\
\hline \multicolumn{4}{|l|}{ Medication } \\
\hline $\begin{array}{l}\text { Acetylsalicylic acid } \\
\text { and/or clopidogrel (\%) }\end{array}$ & 67 (94.7\%) & 74 (97.4\%) & 0.430 \\
\hline Nitrates (\%) & II (I5.5\%) & $16(21.1 \%)$ & 0.404 \\
\hline$\beta$-blockers (\%) & 65 (91.6\%) & $67(88.2 \%)$ & 0.591 \\
\hline $\begin{array}{l}\text { Calcium channel } \\
\text { blockers (\%) }\end{array}$ & II (I5.5\%) & $19(25.0 \%)$ & 0.218 \\
\hline ACEI or ARB (\%) & 45 (63.2\%) & $48(63.2 \%)$ & 1.000 \\
\hline Statins (\%) & $60(84.5 \%)$ & $73(96.1 \%)$ & 0.023 \\
\hline
\end{tabular}

Notes: Values are number of cases (\%) or the mean \pm standard deviation, except for TG, which is expressed as the median (lowest quartile; highest quartile).

Abbreviations: PWVba, brachial-ankle pulse wave velocity; SD, standard deviation; MACE, major cardiovascular adverse event; MI, myocardial infarction; DM, diabetes mellitus; SBP, systolic blood pressure; DBP, diastolic blood pressure; MAP, mean arterial pressure; BMI, body mass index, FBG, fasting blood glucose; TC, serum total cholesterol; TG, triglycerides; ACEl, angiotensin-converting enzyme inhibitor; ARB, angiotensin II receptor blockers.

The role of factors such as smoking, BMI, previous MI, and DM were not found to be significant. Only $12 \%$ of patients were found to have DM and all of these were treated with dietary adjustments. Age at baseline was not correlated with prognosis in this cohort (RR 0.99; 95\% CI:0.96-1.03,
$P=0.787$ ). Six-month change in SBP (RR $0.99 ; 95 \%$ CI:0.67-1.01; $P=0.270$ ), DBP (RR 0.97; 95\% CI:0.94-1.01; $P=0.159)$, PP (RR 0.99; 95\% CI:0.97-1.02; $P=0.600)$, MAP (RR 0.98-1.01; 95\% CI:0.95-1.01; $P=0.171$ ), and blood chemistry were not significant. Also, the type of medication was not found to be predictive of outcome.

To assess the influence of changes in PWVba on occurrence of MACE when considering the contribution of other factors, three models of the Cox proportional hazards regression, with a different set of dependent variables, have been created (Table 4). $\triangle \mathrm{PWVba}$ was not found to influence prognosis, when age at baseline, and changes in MAP at six-month follow-up here applied to the first model (Model 1). In Model 2, we included $\Delta \mathrm{PWVba}, \Delta \mathrm{TC}$, and $\triangle \mathrm{SBP}$ at six months, as well as age and whether the patient was a smoker at the moment of inclusion. After applying adjustments for other studied factors (Model 3), PWVba was still found to have an influence on prognosis (Table 4).

Kaplan-Meier curves show the probabilities of event-free survival of patients with negative $\Delta \mathrm{PWV}$ or positive $\Delta \mathrm{PWV}$ (Figure 1).

\section{Discussion}

Carotid-femoral PWV method is considered to be the "gold standard" for measurement of arterial rigidity. This method has been used in the majority of epidemiological studies demonstrating the predictive value of aortic stiffness for cardiovascular events. In their studies preceding our investigation, Japanese researchers focused on the value of brachial-ankle pulse wave velocity (PWVba) testing and showed the aortic PWV as the primary independent correlate of PWVba. ${ }^{9}$ We employed the PWVba method after considering that it is simple to use, inexpensive, well reproduced, and its informative value has been demonstrated in multiple studies. ${ }^{5-7,10}$ Additionally, we used the PWVba method because it is more comfortable for the average patient,

Table 4 Multivariate Cox proportional hazard regression analyses of MACE $(n=161)$

\begin{tabular}{|c|c|c|c|c|}
\hline & $\mathbf{R} \mathbf{R}$ & $95 \% \mathrm{Cl}$ & $t$-value & $P$-value \\
\hline \multicolumn{5}{|l|}{ Model I $(P=0.004)$} \\
\hline$\Delta \mathrm{PWVba}(\mathrm{I}=$ positive $/ 0=$ negative $)$ adjusted $\Delta \mathrm{MAP}$ and age at baseline & 4.40 & $2.03-9.52$ & 3.17 & 0.002 \\
\hline \multicolumn{5}{|l|}{ Model $2(P=0.007)$} \\
\hline $\begin{array}{l}\Delta \mathrm{PWVba}(\mathrm{I}=\text { positive } / 0=\text { negative }) \text { adjusted } \Delta \mathrm{SBP}, \Delta \mathrm{TC}, \text { current smoking, and age at baseline } \\
\text { Model } 3(P=0.005)\end{array}$ & 4.57 & $2.08-10.06$ & 3.18 & 0.002 \\
\hline $\begin{array}{l}\Delta \mathrm{PWVba}(\mathrm{I}=\text { positive } / 0=\text { negative }) \text { adjusted } \Delta \mathrm{SBP}, \Delta \mathrm{DBP}, \Delta \mathrm{TC} \text {, current smoking and age at } \\
\text { baseline, previous } \mathrm{MI}, \mathrm{DM}, \mathrm{ACE} / \mathrm{ARB} \text { use }\end{array}$ & 3.99 & $\mathrm{I} .8 \mathrm{I}-8.78$ & 2.90 & 0.004 \\
\hline
\end{tabular}

Abbreviations: RR, rate ratio; $\mathrm{Cl}$, confidence intervals; PWVba, brachial-ankle pulse wave velocity; MAP, mean arterial pressure; SBP, systolic blood pressure; TC, total cholesterol; DBP, diastolic blood pressure; MI, myocardial infarction; DM, diabetes mellitus; ACEl, angiotensin-converting-enzyme inhibitor; ARB, angiotensin II receptor blockers. 


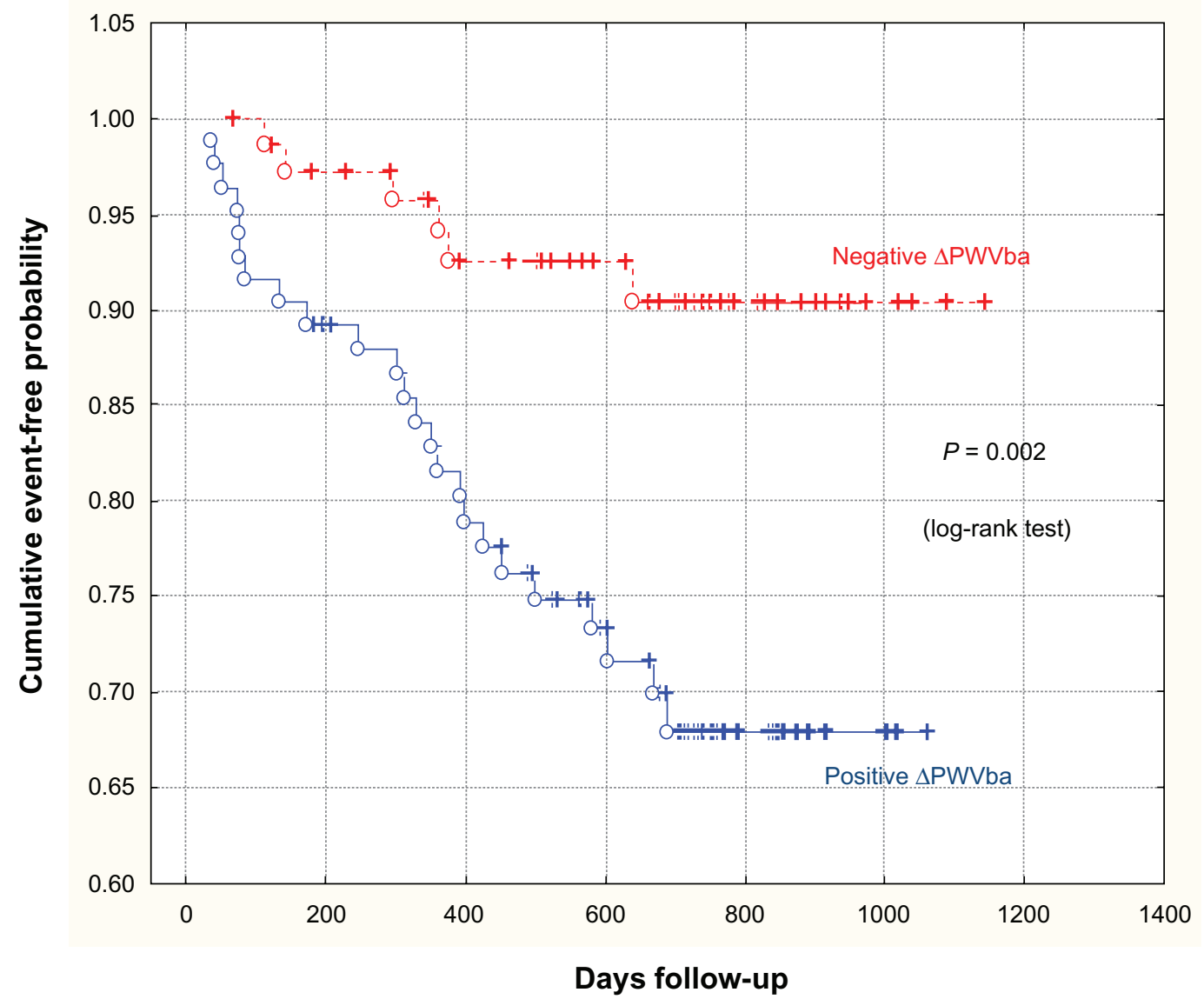

Figure I Probability of event-free survival according to $\triangle \mathrm{PWV}$. The difference between patients with negative $\Delta \mathrm{PWV}$ and patients with positive $\Delta \mathrm{PWV}$ was significant $(P=0.002)$.

since it allows him/her to remain fully dressed during the procedure.

Our own previous publications, as well as those by other investigators, have shown that increased arterial rigidity in patients with CAD is associated with increased risk of MACE. ${ }^{5,4,11}$ The expert consensus on arterial stiffness states that clinical trials need to be conducted in order to determine whether a reduction in arterial stiffness is a desirable therapeutic goal, in terms of hard clinical endpoints such as morbidity and mortality. ${ }^{12}$ In the current study, we aimed to answer the question of whether a decrease in arterial stiffness leads to improved prognosis in male patients suffering from CAD.

Based on the data from the group as a whole, we did not note distinctions in the levels of PWVba, BP, TC, TG, and FBG throughout the term of the study. Regression analysis demonstrated that $\triangle \mathrm{PWVba}$ was significantly correlated with $\triangle \mathrm{BP}$. The closest correlation we found was between $\triangle \mathrm{PWVba}$ and delta MAP $(r=0.36 ; P<0.001)$. In many studies executed on hypertensive and normotensive patients, there is a parallel decrease in BP and PWV as a result of therapy. ${ }^{13,14}$ However, it is important to remember that in and of itself, BP is one of the most meaningful factors of cardiovascular risk and a decrease in BP results in improved prognosis. ${ }^{15}$ The next question is whether reduction of morbidity and mortality in patients with cardiovascular pathology is only due to the influence of therapy on BP, or if some medications possess their own additional influence on the patient's prognosis. In the last few years, there have been a few published studies focusing on some medications which have a "BP-independent" positive influence on stiffness of large arteries and central hemodynamics. ${ }^{16-18}$

The vast majority of patients received massive vasoactive therapy (angiotensin-converting-enzyme inhibitor or angiotensin II receptor blockers about $60 \%$; $\beta$-blockers $>70 \%$; calcium channel blockers and nitrates $>20 \%$ ), both before inclusion and throughout the course of our study. One of the goals of the study was to find out whether variations in arterial stiffness have an influence on the three-year prognosis of patients with $\mathrm{CAD}$, irrespective of change in $\mathrm{BP}$.

As it stands today, only Guerin et al have shown the influence of aortic stiffness attenuation on survival of 
patients (a study of end-stage renal failure patients). ${ }^{19}$ In their study, all patients received antihypertensive therapy at inclusion. From entry until the end of follow-up, the changes in aortic PWV in response to decreased BP were measured using ultrasound. At target BP, patients were separated into two groups based on the direction of change in the PWV. PWV, like in our study, correlated with $\triangle \mathrm{SBP}(r=0.54$; $P<0.0001)$ and was not tied to variations in biochemical parameters or type of applied therapy. Analysis at the end of the study revealed that in the group with $\Delta \mathrm{PWVba} \geq 0$, there was a $70 \%$ mortality rate, while in the other group, this rate was only $26 \%$.

Following the method of Guerin et al ${ }^{19}$ we too separated patients into groups according to the direction of variation in their PWVba in the first six months. For 85 patients, arterial stiffness either remained unchanged, or increased $(\triangle \mathrm{PWVba} \geq 0)$, while it decreased for the other 76 patients $(\triangle \mathrm{PWVba}<0)$. Thus, $80 \%$ of all MACEs occurred in the group with positive PWVba, as compared with $20 \%$ in the negative PWVba group. Based on our data, the sensitivity of PWV was $80 \%$ and its specificity was $54 \%$. For Guerin et al these parameters were $56 \%$ and $84 \%$, respectively. ${ }^{19}$

Univariate Cox analysis showed that the absence of PWVba decrease in the first six months carried a 3.8 times MACE risk increase for these patients. Changes in other risk factors were not associated with improved prognosis. Adjustment for age and $\triangle \mathrm{MAP}$ (Model 1), age and smoking profile at inclusion, and change in BP and TC at six-month follow-up (Model 2), and other factors (Model 3), PWVba was found to have maintained its prognostic predictive value.

With the exception of statins, there were no differences in medication between groups with "positive" and "negative" $\triangle \mathrm{PWVba}$. In the group with "positive" $\triangle \mathrm{PWVba}$ there was a $12 \%$ higher rate of statin use than in the "negative" $\Delta \mathrm{PWVba}$ group $(P=0.023)$.

It should be noted that patients who showed a decreased PWVba in six months had, on average, higher values of this parameter at inclusion in the study than patients with an increase in PWVba. In the "negative" $\triangle \mathrm{PWVba}$ group, at inclusion, there were higher values for BP and TC $(P<0.05)$. Taking these facts into account, at baseline, patients showing a decrease in arterial stiffness seem to be at higher risk of MACE. Also, patients who in the first six months of therapy were found to have an increased or unchanged PWVba, likely had more advanced vascular lesions which were either irreversible or required a more prolonged period of treatment.
Many authors, when discussing pharmacological influence on arterial rigidity, separate "acute" effects from "chronic" effects. Acute effects are to a greater degree connected with reduced vascular tone, while chronic effects are mediated through structural modifications of the arterial wall. ${ }^{20,21}$ In various studies, positive vascular remodeling was evaluated after 6-9 months or more, and was not dependent upon other risk factors. ${ }^{16,18,22}$ These and other works have allowed for the 2006 Expert Consensus document on arterial stiffness to conclude that arterial stiffness attenuation may reflect the true reduction of arterial wall damage, whereas BP, glycemia, and lipids can be normalized in a few weeks by using antihypertensive, anti diabetic, and lipid-lowering drugs, leading to a strong reduction in $\mathrm{CV}$ risk scores, but without any improvement yet of atherosclerotic lesions and arterial stiffness, which requires a long-lasting correction of biochemical abnormalities. ${ }^{12}$ Therefore, it can be supposed that a therapeutic strategy, having the goal of reducing arterial stiffness, can lead to more significant improvement of prognosis than correction of individual risk factors.

\section{Conclusion}

Decreased PWVba on a six-month background of treatment is an independent predictor of favorable prognosis in the next three years for men with CAD. Our results suggest that for patients with $\mathrm{CAD}$, on a long treatment background, reduction of rigidity in large arteries may appear to be a more reliable criterion of therapeutic efficacy than the dynamics of conventional cardiovascular risk factors.

\section{Acknowledgment}

This study was supported by Mr Alexander Shapeton of University of Massachusetts Medical School.

\section{Disclosure}

The authors report no conflicts of interest in this work.

\section{References}

1. Blacher J, Guerin AP, Pannier B, Marchais SJ, Safar ME, London GM. Impact of aortic stiffness on survival in end-stage renal disease. Circulation. 1999;99:2434-2439.

2. Laurent S, Boutouyrie P, Asmar R, et al. Aortic stiffness is an independent predictor of all-cause and cardiovascular mortality in hypertensive patients. Hypertension. 2001;37:1236-1241.

3. Cruickshank K, Riste L, Anderson SG, Wright JS, Dunn G, Gosling RG. Aortic pulse-wave velocity and its relationship to mortality in diabetes and glucose intolerance: an integrated index of vascular function? Circulation. 2002;106:2085-2090.

4. Stefanadis C, Dernellis J, Tsiamis E, et al. Aortic stiffness as a risk factor for recurrent acute coronary events in patients with ischemic heart disease. Eur Heart J. 2000;21:390-396. 
5. Tomiyama H, Koji Y, Yambe M, et al. Brachial-ankle pulse wave velocity is a simple and independent predictor of prognosis in patients with acute coronary syndrome. Circ J. 2005;69:815-822.

6. Yamashina A, Tomiyama $\mathrm{H}$, Takeda $\mathrm{K}$, et al. Validity, reproducibility, and clinical significance of noninvasive brachial-ankle pulse wave velocity measurement. Hypertens Res. 2002;25:359-364.

7. Liu H, Yambe T, Zhang X. Comparison of brachial-ankle pulse wave velocity in Japanese and Russians. Tohoku J Exp Med. 2005;207: 263-270.

8. van Bortel LM, Duprez D, Starmans-Kool MJ, et al. Clinical applications of arterial stiffness, Task Force III: recommendations for user procedures. Am J Hypertens. 2002;15:445-452.

9. Sugawara J, Hayashi K, Yokoi T, et al. Brachial-ankle pulse wave velocity: an index of central arterial stiffness? J Hum Hypertens. 2005; 19:401-406.

10. Imanishi R, Seto S, Toda G, et al. High brachial-ankle pulse wave velocity is an independent predictor of the presence of coronary artery disease in men. Hypertens Res. 2004;27:71-78.

11. Orlova IaA, Kuz'mina AE, Masenko VP, Iarovaia EB, Ageev FT. Effect of arterial stiffness on development of cardio-vascular complications in ischemic heart disease. Kardiologiia. 2009;49: 11-17.

12. Laurent S, Cockcroft J, van Bortel L, et al; for European Network for Non-invasive Investigation of Large Arteries. Expert consensus document on arterial stiffness: methodological issues and clinical applications. Eur Heart J. 2006;27:2588-2605.

13. Asmar R, Topouchian J, Pannier B, Benetos A, Safar M; for Scientific, Quality Control, Coordination and Investigation Committees of the Complior Study. Pulse wave velocity as endpoint in large-scale intervention trial. The Complior study. Scientific, Quality Control, Coordination and Investigation Committees of the Complior Study. J Hypertens. 2001;19:813-818.
14. Kim KH, Jeong MH, Cho SH, et al. Clinical effects of calcium channel blocker and Angiotensin converting enzyme inhibitor on endothelial function and arterial stiffness in patients with angina pectoris. $J$ Korean Med Sci. 2009;24:223-231.

15. Staessen JA, Wang JG, Thijs L. Cardiovascular protection and blood pressure reduction: a meta-analysis. Lancet. 2001;358:1305-1315.

16. Tropeano AI, Boutouyrie P, Pannier B, et al. Brachial pressureindependent reduction in carotid stiffness after long-term angiotensinconverting enzyme inhibition in diabetic hypertensives. Hypertension. 2006;48:80-86.

17. Karalliedde J, Smith A, deAngelis L, et al. Valsartan improves arterial stiffness in type 2 diabetes independently of blood pressure lowering. Hypertension. 2008;51:1-7.

18. Protogerou A, Blacher J, Stergiou GS, Achimastos A, Safar ME. Blood pressure response under chronic antihypertensive drug therapy: the role of aortic stiffness in the REASON (Preterax in Regression of Arterial Stiffness in a Controlled Double-Blind) study. J Am Coll Cardiol. 2009; 53:445-451.

19. Guerin AP, Blacher J, Pannier B, Marchais SJ, Safar ME, London GM. Impact of aortic stiffness attenuation on survival of patients in end-stage renal failure. Circulation. 2001;103:987-992.

20. Laurent S, Boutouyrie P, Lacolley P. Structural and genetic bases of arterial stiffness. Hypertension. 2005;45:1050-1055.

21. Lacolley P, Ghodsi N, Glazer E, et al. Influence of graded changes in vasomotor tone on the carotid arterial mechanics in live spontaneously hypertensive rats. Br J Pharmacol. 1995;115:1235-1244.

22. Boutouyrie P, Bussy C, Hayoz D, et al. Local pulse pressure and regression of arterial wall hypertrophy during long-term antihypertensive treatment. Circulation. 2000;101:2601-2606.
Vascular Health and Risk Management

\section{Publish your work in this journal}

Vascular Health and Risk Management is an international, peerreviewed journal of therapeutics and risk management, focusing on concise rapid reporting of clinical studies on the processes involved in the maintenance of vascular health; the monitoring, prevention and treatment of vascular disease and its sequelae; and the involvement of

\section{Dovepress}

metabolic disorders, particularly diabetes. This journal is indexed on PubMed Central and MedLine. The manuscript management system is completely online and includes a very quick and fair peer-review system, which is all easy to use. Visit http://www.dovepress.com/ testimonials.php to read real quotes from published authors. 